\title{
FULL PAPER \\ Generation of a functional, soluble tapasin protein from an alternatively spliced mRNA
}

\author{
B Gao ${ }^{1}$, A Williams ${ }^{2}$, A Sewell ${ }^{3}$ and T Elliott ${ }^{2}$ \\ ${ }^{1}$ Rheumatology Unit, Institute of Child Health, University College London, UK; ${ }^{2}$ Cancer Sciences Division, University of Southampton \\ School of Medicine, Southampton, UK; ${ }^{3}$ Nuffield Department of Clinical Medicine, University of Oxford, John Radcliffe Hospital, UK
}

\begin{abstract}
The loading of newly synthesised $\mathrm{MHC}$ class I molecules $(\mathrm{MHCl})$ with peptides requires the involvement of several endoplasmic reticulum (ER)-resident cofactors including calnexin, calreticulin, transporter associated with antigen processing, ERp57 and tapasin. In the absence of tapasin, MHC I complexes are loaded with suboptimal peptides and their recognition by cytotoxic $T$ cells raised to high-affinity, immunodominant peptide epitopes is impaired. Here, we describe the cloning and functional assessment of an alternative spliced form of tapasin. From the EST database, we obtained a partially spliced tapasin cDNA that retained introns 4-6. When transfected into the tapasin-deficient cell line 0.220, the cDNA produced an alternatively spliced tapasin transcript that contained intron 5 (74 bp). This introduced a new stop codon that terminated translation immediately before the putative transmembrane domain and led to a tapasin molecule containing the lumenal domain plus 8 extra novel amino acids at its C-terminus. This molecule promoted peptide loading of HLA-B5 in 0.220 cell line, and restored normal HLA-B5 surface expression. However, the peptides loaded onto HLA-B5 were suboptimal compared to those loaded onto HLA-B5 in the presence of wild-type tapasin.
\end{abstract}

Genes and Immunity (2004) 5, 101-108. doi:10.1038/sj.gene.6364043

Published online 11 December 2003

Keywords: tapasin; antigen processing; MHC class I assembly

\section{Introduction}

MHC class I complexes comprise a polymorphic, glycosylated, transmembrane heavy chain (HC), a noncovalently associated beta-2-microglobulin $(\beta 2-\mathrm{m})$ subunit and a short peptide, usually of 8-10 amino acids. ${ }^{1}$ These complexes are expressed on the surface of virtually all nucleated cells and provide a means of discriminating self from nonself through recognition by cytotoxic T cells (CTLs) and natural killer (NK) cells. Most peptides that bind to MHC class I molecules are derived from a fraction of newly but aberrantly synthesised proteins that are targeted for cytosolic degradation. $^{2,3}$ The peptides generated are transported by the peptide transporter associated with antigen processing (TAP) into the endoplasmic reticulum (ER), where they are loaded onto peptide-receptive MHC class I complexes. The peptide-loaded MHC class I complex is then located to the cell surface for subsequent recognition.

The assembly of MHC class I molecules with peptides that are able to stabilise the interaction between $\mathrm{HC}$ and $\beta 2-\mathrm{m}$ is a critical event in antigen presentation and involves at least six ER-resident cofactors: calnexin, calreticulin, ERp57, TAP, tapasin and ERAAP.-10 The first five of these associate to form the peptide-loading

Correspondence: Dr T Elliott, Cancer Sciences Division, University of Southampton School of Medicine, Mailpoint 824, Somers Cancer Research Building, Southampton General Hospital, Tremona Road, Southampton SO166YD, UK. E-mail: tje@soton.ac.uk

Received 15 April 2003; revised 20 October 2003; accepted 20 October 2003 complex (PLC), which is believed to promote the assembly of peptide-receptive complexes with peptide within the ER. Unlike calreticulin, calnexin and ERp57, which have general chaperone functions, tapasin has a specialised role in MHC class I assembly. ${ }^{11}$ It mediates an interaction between TAP and newly synthesised MHC class I molecules through two functional sites; one binds to and stabilises the TAP heterodimer and the other associates to the peptide-receptive MHC class I complex. ${ }^{12}$ The TAP protein is more stable when associated with tapasin, and peptide binding to TAP and translocation of peptides into the ER are increased when tapasin is present. Tapasin also plays an active role in the association of peptide-receptive MHC class I complexes with the other ER cofactors and is absolutely required for the association of MHC class I complexes with ERp57 and calreticulin within the PLC. ${ }^{13}$ It also enhances the rate and extent of class I MHC loading with optimal peptides. ${ }^{14-17}$ In tapasin-deficient cells, the repertoire of class I-bound peptide is thus skewed towards less stably binding ones, a phenomenon that is consistent with the phenotype of C57BL/6 mice that lack tapasin and B2705 expressed in $0.220 .^{18-20}$

Tapasin is a type I transmembrane glycoprotein with a dilysine ER-retention motif $(\mathrm{K} \times \mathrm{K} x \mathrm{x})$ in its cytoplasmic tail. The tapasin gene consists of eight exons within a gene cluster approximately $190 \mathrm{~kb}$ centromeric to the HLA-DPB2 locus. The leader sequence and the prolinerich N-terminal portion of the lumenal domain are encoded by the first four exons. Exon 5 encodes the remainder of the lumenal domain, having homology to the immunoglobulin superfamily. Exon 6 encodes a 
putative transmembrane domain and exons 7 and 8 encode a short cytoplasmic domain that contains the ER-retention motif. ${ }^{21}$ The TAP-binding site has been localised to exons 6-8. ${ }^{12}$ Accordingly, an engineered construct of tapasin comprising its lumenal domains is secreted and has been shown to restore the antigenpresenting function of HLA-B8 in a tapasin-negative cell line in the absence of HLA-B8 association with TAP. ${ }^{22}$ The N-terminal 50 amino acids of tapasin (encoded within exon 2) are essential for its interaction with class I MHC, CRT and Erp57, while a mutant mouse tapasin lacking amino acids 334-342 (encoded within exon 5) fails to bind to a mouse class I allele, but surprisingly, facilitates its assembly and expression. ${ }^{23}$ We show here that an alternatively spliced form of the tapasin gene that encodes a truncated tapasin containing only the lumenal domains of tapasin plus eight novel C-terminal residues (KSWELCGI) is functional in terms of its ability to promote optimal peptide loading, restore class I surface expression and support a CTL response.

\section{Results}

An alternatively spliced tapasin gene (T5) encodes a soluble form of tapasin

The published sequence of tapasin (GenBank, accession AF009510) was used to search the GenBank database of expressed tag sequences derived from a large panel of cDNA libraries, using the TBLASTN local alignment search tool. ${ }^{24}$ Several cDNAs were identified as having a high degree of homology to tapasin and were acquired from the UK HGMP resource centre (www.hgmp.mrc. ac.uk) and sequenced. One of the clones revealed a 2202 bp sequence (Genbank, accession AF067286) that was identical to the published cDNA sequence over the first 1215 nucleotides, and thereafter was identical to the genomic sequence, having retained introns 4-7 (T5, Figure 1). A stop codon was identified within intron 4, resulting in a transcript that was predicted to encode a short version of tapasin including the proline-rich $\mathrm{N}$ terminal region of the molecule, but lacking the Ig SF domain, transmembrane domain and cytoplasmic domain. To determine whether this truncated gene was functional, it was transfected it into the tapasin-defective B cell line 0.220-B5.

Surprisingly, RT-PCR analysis of the transfected cells with a primer pair spanning exons 4 and 6 gave a $200 \mathrm{bp}$ product that was shorter than the expected product derived from the transfected gene $(\sim 500 \mathrm{bp})$, but longer than that expected for wild-type tapasin (Figure 2a). This suggested that mRNA from the transfected cDNA was processed in such a way as to excise intron 4 but retain intron 5. We confirmed this using primers spanning intron 5 (Figure 2b). A band of $194 \mathrm{bp}$ was seen in $0.220-$ B5T5 (lane 5) corresponding to $78 \mathrm{bp}$ of intron 5 plus $116 \mathrm{bp}$ of flanking sequence. The latter $116 \mathrm{bp}$ product was also seen in the nontransfected 0.220-B5 (lane 6), as 0.220 does possess genomic tapasin DNA but does not express a functional tapasin protein due to a point mutation in the splice-acceptor site of exon 2. ${ }^{25}$ These data suggested that the constitutive mutant gene is otherwise processed normally-an observation that is consistent with the shortened tapasin seen in these cells. Interestingly, a minor RT-PCR product with retained intron 5 was also found in all control cell lines, suggesting that the alternatively spliced form may be generated in vivo from a naturally produced transcript. We assume that the low level of detectable 'wild-type' tapasin products in LBL721.220-B5T5 in Figure 2b is due to competition for transcription factors between the endogenous gene and the highly expressed transgene. Figure $2 \mathrm{~b}$ also shows that both wild-type tapasin and a transcript including intron 5 are expressed in peripheral blood lymphocyte (PBL), the latter being the least abundant. The level of each RT-PCR product did not change after the activation of PBL with either $75 \mu \mathrm{g} / \mathrm{ml}$ conA, $10 \mu \mathrm{g} \mathrm{ml} \mathrm{PHA} \mathrm{or} 5 \mu \mathrm{g} / \mathrm{ml}$ PWM (data not shown). To confirm the nature of the major tapasin transcript expressed in 0.220-B5T5, we cloned and sequenced the RT-PCR product from B5T5 using primers flanking exons 4 and 6 (Figure 2a, lane 2). The sequencing results showed that intron 4 was indeed spliced out, while intron 5 was retained as part of the transcript (data not shown, Figure 2c). As expected, 220-B5T5 cells expressed a tapasin protein that was slightly smaller $(45 \mathrm{kDa})$ than wild-type tapasin (48 kDa) (Figure 3a). When T5 cDNA was translated in vitro, where no further processing of T5 transcripts can occur, the expected product of around $35 \mathrm{kDa}$ was generated corresponding to exons 1-4 (Figure 3a, lane 7). Western blot analysis of various cell lines was carried out in an attempt to identify either of these tapasin splice variants at the protein level. Figure $3 b$ shows that human monocyte-derived dendritic cell lines of both immature and mature phenotype do not express any altered tapasin protein. Similarly, when a panel of tissue-specific protein blots were probed with the antitapasin antisera only the full-length form was seen in the spleen, lung and placenta, with little tapasin protein identified in uterus, brain and kidney lysates. Figure $3 c$ shows the addition of the eight novel amino acids (KSWELCGI) that are in-frame with the terminal bases of the preceeding exon 5. The full-length tapasin would include a further 44 amino acids encoded from exons 6-8. Therefore, the $3 \mathrm{kDa}$ difference between the two tapasin forms relates to the 36 amino-acid truncation of the full-length tapasin form. The truncated T5-derived tapasin is likely to be secreted, since an analogous soluble construct of tapasin comprising exons 1-5 with eight amino acids from exon 6 is secreted when transfected into 0.220 (data not shown).

\section{Alternatively spliced tapasin restores class I surface expression and rescues the presentation of HIV-gag epitope in 0.220-B8 cell line to a B8-restricted CTL}

Many MHC class I alleles are expressed at low levels at the cell surface when transfected into the tapasindeficient cell LBL721.220 as a result of their inefficient assembly with peptides. ${ }^{26,27}$ Figure 4 a shows that the cell surface expression of HLA-B5 is lower in 721.220 compared to that of class I in the tapasin-competent cell line 221.A24. However, surface HLA-B5 expression is restored by transfection of the $\mathrm{T} 5 \mathrm{cDNA}$, to a level approaching MHC class I expression seen in 221-A24. A similar result was obtained for HLA-B8 (data not shown). This indicates that the alternatively spliced gene product is able to promote the loading of class I MHC with peptides.

The apparent restoration of HLA-B5 surface expression by tapasin T5 led us to determine whether the 
Native_Tpn

T5_Tpn

Native_Tpn

T5_Tpn

Genomic_Tpn

Native_Tpn

Native_Tpn
T5_Tpn
Genomic_TP

Native_Tpn

T5_Tpn

Genomic_Tpn

Native_Ten

T5_Tpn

Genomic_Tpn

Native_Tpn

T5_Tpn

Native_Tpn

T5_Tpn

Native_Tpn

T5_Tpn

Genomic_Tpn

Native_Tpn

T5_Tpn

Native_Tpn

T5 The - Ton

Native_Tpn

'T5_I'pn

Genomic_Tpn

Native_Tpn

T5_Tpn

Genomic_Tpn

Native_Tpn

T5_Tpn

Genomic_Tpn

Native_Tpn

T5_Tpn

Genomic_Tpn

Native_Tpn

T5_Tpn

Genomic_Tpn

Native_Tpn

T5_Ten

Genomic_Tpn

Native_Tpn

T5_Tpn

Genomic_Tpn

Native_Tpn

T5 Tpm

Genomic_Tpn

Native_Tpn

T5_Tpn

Genomic_Tpn

Native_Tpn

T5_Tpn

Genomic_'t'pn

Native_Tpn

T5_Tpn

Genomic_Tpn

Native_Tpn

T5_Tpr

Genomic. Ten

Native_Tron

T5_Tpn

Genomic_Tpn

ATGAAGTCCCTGTCTCTGCTCCTCGCTGTGGCTTTGG

-17040] ATGAAATCCCTGTCTCTGCTCCTGGCTGTGGCTPTGG

de

GCCTGGCGACCGCCGTCTCAGCAGGACCCGCGGTGATCGAGTGTTGGTTCGTGGAGG

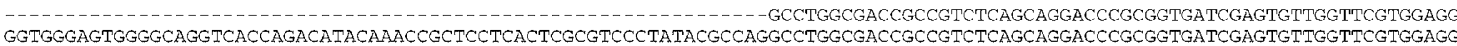

ATGCGAGCGGAAAGGGCCTGGCCAAGAGACCCGGTGCACTGCTGTTGCGCCAGGGACCGGGGGACCGCCGCCCCGGCCGGACCTCGACCCTGAGCTCTATCTCAGTGTACACGATGCGAGGGAAAGGCCCTGCCCAAGAGACCCGTGCACTGCTGTTGCGCCAGGACCGGGGAACCGCCGCCCCGGCCGGACCTCGACCCTGAGCTCTATCTCAGTGTACACG-I-IATGCGAGCGGAAAGGGCCTGGCCAAGAGACCCGGTGCACTGCTGTTGCGCCAGGGACCGGGGGAACCGCCGCCCCGGCCGGACCTCGACCCTGAGCTCTATCTCAGTGTACACGGTGAGT

CTCTAGGGACTCGCGCCCCCCTACCTCTGTCGCCTCCACCGAAACCCCCTCCTCTTTAGATCCGGCAGTGACCTCAGGCCTCAGCTTCCCCTTTGTAAAGTGAGTCTCACTACGGGGTA GTCTGTGCATPTGAAGTTCCCCGAACGCTGCCCTTCCAGCCCCTTTCCCGGCGGTGACTCTACAGCTGCAACTTCCTTCTCTACACTCAGACCCCGCGGGCGCCCTCCAGGCTGCCTTCA

GGCGGTÄTCCCCGGGGGCCCCCGCACCACACTGCGAGATGAGCCGCTTCGTGCCTCTCCCCGCCTCTGCGAAATGGGCCAGCGGCCTGACCCCGCGCAGAACTGCCCGCGGGCCCTG GGCGGTATCCCCGGGGCGCCCCCGCACCACACTGCGAGATGAGCCGCTTCGTGCCTCTCCCCGCCTCTGCGAAATGGGCCAGCGGCCTGACCCCCGCGCAGAACTGCCCGCGGGCCCTGG GGCGGTATCCCCGGGGCGCCCCCGCACCACACTGCGAGATGAGCCGCTTCGTGCCTCTCCCCGCCTCTGCGAAATGGGCCAGCGGCCTGACCCCCGCGCAGAACTGCCCGCGGGCCCTGG

ATGGGGCTTGGCTGATGGTCAGCATATCCAGCCAGTCCTCAGCCTCTCCAGCCTCTTGCGACCACAGCCAGAGCCTCAGCAGGAGCCTGTTCTCATCACCATGGCAACAG--- -- / / ATGGGGCTTGGCTGATGGTCAGCATATCCAGCCCAGTCCTCAGCCTCTCCAGCCTCTTGCGACCACAGCCAGAGCCTCAGCAGGAGCCTGTTCTCATCACCATGGCAACAG--1--1//-

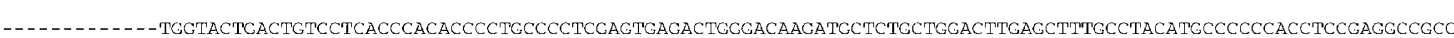
. TGGTACTGACTGTCCTCACCCACACCCCTGCCCTCGAGTGAGACTGGGACAAGATGCTCTGCTGGACTTGAGCTTTGCCTACATGCCCCCCACCTCCGAGGCCGCC -----[15694] TGGTACTGACTGTCCTCACCCACACCCCTGCCCCTCGAGTGAGACTGGGACAAGATGCTCTGCTGGACTTGAGCTTTGCCTACATGCCCCCCACCTCCGAGGCCGCC

TCATCTCTGGCTCCGGGTCCCCCTCCCTTTGGGCTAGAGTGGCGACGCCAGCACCTGGGTAAGGGACATCTGCTCCTGGCTGCAACTCCTGGGCTGAATGGCCAGATGCCAGCAGCCCAA TCATCTCTGGCTCCGGGTCCCCCTCCCTTTGGGCTAGAGTGGCGACGCCAGCACCTGGGTAAGGGACATCTGCTCCTGGCTGCAACTCCTGGGCTGAATGGCCAGATGCCAGCAGCCCAA TCATCTCTGGCTCCGGGTCCCCCTCCCTTTGGGCTAGAGTGGCGACGCCAGCACCTGGGTAAGGGACATCTGCTCCTGGCTGCAACTCCTGGGCTGAATGGCCAGATGCCAGCAGCCCAA

GAAGGGGCGTGGCATTTGCTGCTTGGGATGATGATGAGCCATGGGGCCCATGGACCGGAAATGGGACCTTCTGGCTGCCTAGAGTTCAACCCTTTCAGGAGGGCACCTATCTGGCCACC GAAGGGGCCGTGGCATTTGCTGCTTGGGATGATGATGAGCCATGGGGCCCATGGACCGGAAATGGGACCTTCTGGCTGCCTAGAGTTCAACCCTTTCAGGAGGGCACCTATCTGGCCACC GAAGGGGCCGTGGCATTTGCTGCTTGGGATGATGATGAGCCATGGGGCCCATGGACCGGAAATGGGACCTTCTGGCTGCCTACAGTTCAACCCTTTCAGGAGGGCACCTATCTGGCCACO

ATACACCTGCCATACCTGCAAGGACAGGTCACCCTGGAGCTTGCTGTGTACA

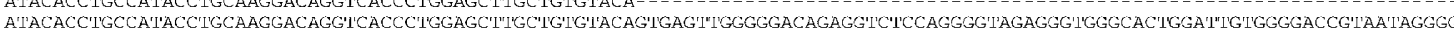
ATACACCTGCCATACCTGCAAGGACAGGTCACCCTGGAGCTTGCTGTGTACAGTGAGTTGGGGGACAGAGGTCTCCAGGGGTAGAGGGTGGGCACTGGATTGTGGGGACCGTAATAGGGO

GAGGGATGATGGATAAGAGGTGCCTGGGCAAGAAGTAGAGATAGAAAGAGGTTCCTGGGAGTAGAGGGGTAATGGGAGGCTAGAAGTTTCCTGGAAATTTGAGGGGTTTGACATGG GAGGGATGATGGATAAGAGGGTGCCTGGGCAAGTAAGTAGAGATAGAAAGAGGTTCCTGGGAGTTAGAGGGGTAATGGGAGGCTAGAAGTTTCCTGGAAATTIGAGGGGCTTTGACATGG

GTATTTCTGTGACGCACCAATGGAGAGACAGTGGGTTCCCTATTTCAGGAGAAGAAACCTAACCTTCTTTAGTTCTGAGGAAGCCAGCAGGCAAACTGAGGGTCTCTTAGGGAGGACAGT GTATTCTGTGCGCACCAATGAGAGACAGTGGTTCCCTATTTAGGAGAAGAAACTAACCTTCTTAGTTCTGAGGAGCCAGCAGGCAACTGAGGGTTCTTAGGGAGGACAGT

AACCCCCCAAAGTGTCCCTGATGCCAGCAACCCTTGCACGGGCCGCCCCAGGGGAGGCACCCCCGGAATTGCTCTGCC ATGGACTGATTTCCCTATGCTCATTTCGTCCTCTTTCCCCAGAACCCCCCAAAGTGTCCCTGATGCCAGCAACCCTTGCACGGGCCGCCCCAGGGGAGGCACCCCCGGAATTGCTCTGCC ATGGACTGATTTCCCTATGCTCATTTCGTCCTCTTTCCCCAGAACCCCCCAAAGTGTCCCTGATGCCAGCAACCCTTGCACGGGCCGCCCCAGGGGAGGCACCCCCGGAATTGCTCTGCO

TTGTGTCCCACTTCTACCCTTCTGGGGGCCTGGAGGTGGAGTGGGNACTCCGGGGTGGCCCAGGGGGCCGCTCTCAGAAGGCCGAGGGGCAGAGGTGGCTCTCGGCCCTGCGCCACCNTI TTGTGTCCCACTTCTACCCTTCTGGGGGCCTGGAGGTGGAGTGGGAACTCCGGGGTGGCCCAGGGGGCCGCTCTCAGAAGGCCGAGGGGCAGAGGTGGCTCTCGGCCCTGCGCCACCATI TTGTGTCCCACTTCTACCCTTCTGGGGGCCTGGAGGTGGAGTGGGAACTCCGGGGTGGCCCAGGGGGCCGCTCTCAGAAGGCCGAGGGGCAGAGGTGGCTCTCGGCCCTGCGCCACCATT

CCGATGGCTCTGTCAGCCTCTCTGGGCACTTGCAGCCGCCCCCAGTCACCACTGAGCAGCATGGGGCACGCTATGCCTGTCGAATTCACCATCCCAGCCTGCCTGCCTCGGGGCGCAGCG CCGATGGCTCTGTCAGCCTCTCTGGGCACTTGCAGCCGCCCCCAGTCACCACTGAGCAGCATGGGGCACGCTATGCCTGTCGAATTCACCATCCCAGCCTGCCTGCCTCGGGGCGCAGCG CCGATGGCTCTGTCAGCCTCTCTGGGCACTTGCAGCCGCCCCCAGTCACCACTGAGCAGCATGGGGCACGCTATGCCTGTCGAATTCACCATCCCAGCCTGCCTGCCTCGGGGCGCAGCG

CTGAGGTCACCCTGGAGGTAGCAG-

CTGAGGTCACCCTGGAGGTAGCAGGTAAGAGCTGGGAGCTCTGCGGATCTGAGCCAGCACCCAGGAAGACAGGAGCTCACTCTCACCCCTTCTGCCTGCCAGGTCTTTCAGGGCCTCC CTGAGGTCACCCTGGAGGTAGCAGGTAAGAGCTGGGAGCTCTGCGGAATCTGAGCCAGCACCCAGGAAGACAGGAGCTCACTCTCACCCCTTCTGCCTGCCAGGTCTTTCAGGGCCCTCC

CTTGAGGACAGCGTAGGCCTTTTCCTGTCTGCCTTTCTTCTGCTTGGGCTCTTCAAGGCACTGGGCTGGGCTG-CTTGAGGACAGTGTAGGCCTITTCCTGTCTGCCTTTCTTCTGCTTGGGCTCTTCAAGGCACTGGGCTGGGCTGGTAAGTGTCAGCCCTACCCTGACCATGACCTGAGGTTGGTGGACTTT CITGAGGACAGCGTAGGCCTITTCCTGTCTGCCTTTCTTCTGCTTGGGCTCTTCAAGGCACTGGGCTGGGCTGGTAAGTGTCAGCCCTACCCTGACCATGACCTGAGGTIGGTGGACTTI

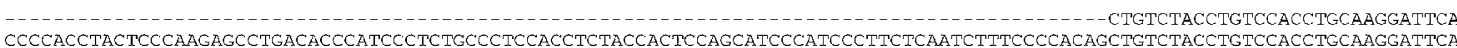
CCCCACCTACTCCCAAGAGCCTGACACCCATCCCTCTGCCCTCCACCTCTACCACTCCAGCATCCCATCCCTTCTCAATCTTTCCCCACAGCTGTCTACCTGTCCACCTGCAAGGATTCA
CCCCACCTACTCCCAAGAGCCTGACACCCATCCCTCTGCCCTCCACCTCTACCACTCCAGCATCCCATCCCTTCTCAATCTTTCCCACAGCTGTCTACCTGTCCACCTGCAAGGATTCA AAGAAG-

AAGAAGGTACAGTGCTCCACCTCTCTGTATCTTTCCCTTGTCACTTTATCTCCTCATCCTATCTCAAAACCCATGGAGGGAGGCTGCTGGTGTGGTAGGCAGAACCTAGGCTTGGAATTC

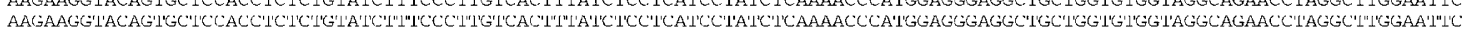

ACACTGATCTGGGTTAAAACCTGGCACTATATCCTAACTGTAGGACTCTTTGAGCATGCTACTTAATCTATATGTTTCCTTGGGTGTAGGGATTTAAAAAGTTACTTAGGCAGTGCTGT ACACTGATCTGGGTTAAAACCTGGCACTATATCCTAACTGTAGGACTCTTTGAGCATGCTACTTAATCTATATGTTTCCTTGGGTGTAGGGATTTTAAAAAGTTACTTAGGCAGTGCTGT

(

CCAGTAGAAGATAATGCAAGCACATATGTAAATTAAAATACTCTAGTACTCACATT- 17418$]-1 / 1,[19307]$ AAAGCAGAGTGAGGGACTCACTGCCATCCTGTGGAAGCCACC

ATCATCTCTGGCCCAAGCTTCTGTAGTAG

ATCATCTCTGGCCCAAGCTTCTGTAGTAG [19380]

Figure 1 Alternatively spliced tapasin (T5). Comparison of the structure of mRNAs T5 and native tapasin with the genomic structure of tapasin. The genomic sequence of the tapasin gene, based on the sequence of cosmid F0811 (Genbank accession number Z971840), was aligned with the sequences of native tapasin (AF009519) and alternatively spliced tapasin (AF067286). Stop codons in introns 4 and 5 are marked in bold type.

antigen-presenting function of class $\mathrm{I}$ molecules is restored by this protein. HLA-B8 is one of the class I MHC alleles whose surface expression is reduced by the absence of tapasin. As shown in Figure 4b, although $0.220-\mathrm{B} 8$ is able to present exogenously added synthetic peptide to B8-restricted CTL, it is unable to present the HIV-1-gag epitope SLYNTVATL from an endogenous source (ie after vaccinia infection). However, presentation of the endogenous epitope was restored in 0.220-B8 transfected with T5. 
104

a

b

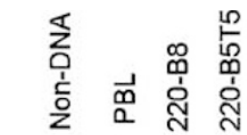

1
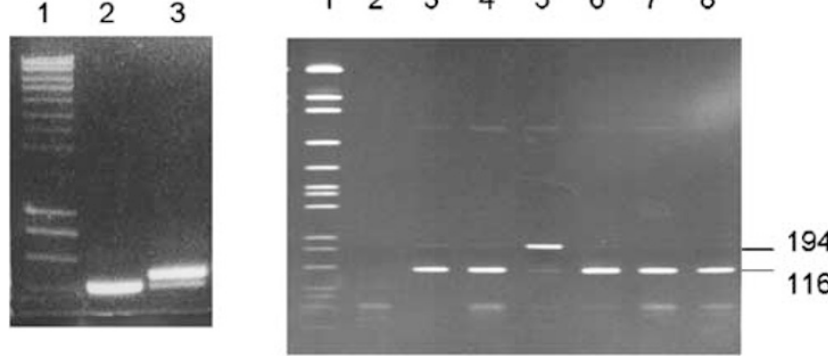

C

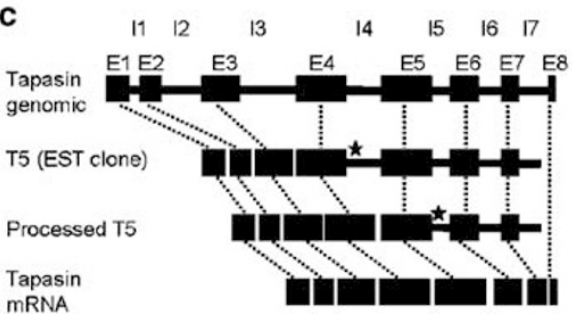

Figure 2 Expression of truncated tapasin in 0.220-B5T5 transfectants. (a) PCR using template cDNA from 220-B5 (lane 2) and 220B5T5 (lane 3) with a primer pair spanning introns 4 and 5. (b) PCR using template cDNA prepared from PBL (lane 3), 220-B8 (lane 4), 0.220-B5T5 (lane 5), 221 (lane 7) and 221-A24 (lane 8) with a primer pair spanning intron 5 . The band at $194 \mathrm{bp}$ corresponds to the expected product from $\mathrm{T} 5$ transcript and the band at $116 \mathrm{bp}$ from the wild-type tapasin. Lane 2 is the negative control for RT and PCR without added RNA. (c) Schematic diagram comparing the intron/ exon organisation of the $\mathrm{T} 5$ transcript (generated by in vitro translation) and the processed T5 transcript (generated by transfection into B cells) with native, full-length tapasin (genomic, top; mRNA, bottom). Stars indicate the position of the most $5^{\prime}$ translation stop codons in $\mathrm{T} 5$ and processed $\mathrm{T} 5$.

\section{T5-encoded tapasin does not associate with TAP}

In order to confirm that the truncated tapasin did not associate with TAP due to the absence of a transmembrane and cytoplasmic domain, we looked for the coimmunprecipitation of the $45 \mathrm{kDa}$ tapasin with TAP1. Figure 5a shows that full-length tapasin $(48 \mathrm{kDa})$ is readily co-precipitated by an anti-TAP1 antibody in immunocompetent LBL 221.45 cells, while in the 220B5T5 transfectant no coassociating tapasin is seen. The truncated protein could be detected in the cell lysate prior to immunoprecipitation (see Figure 3a, lane 5). The results indicate that alternatively spliced tapasin fails to associate with TAP, which confirms previous reports using deliberately engineered soluble tapasin constructs. Consequently, very little $\beta 2-\mathrm{m}$ (a surrogate marker for class I MHC) was seen to coprecipitate with anti-TAP consistent with the MHC class I bridging function of tapasin (Figure $5 b$ ).

\section{T5-encoded tapasin promotes peptide loading and} restores normal trafficking rate of $B 5$ in $\mathbf{0 . 2 2 0}$ cells To investigate the effects of T5-encoded tapasin on the peptide loading and trafficking of HLA-B5, pulse chase experiments were carried out. Pulse-radiolabelled lysates were incubated overnight at $4^{\circ} \mathrm{C}$ to permit dissociation of suboptimally loaded complexes prior to immunoprecipitation with the conformationally sensitive antibody W6/32. The majority of HLA-B5 synthesised in 0.220 were consistent with this phenotype leading to an insufficient number of W6/32 molecules for visualisation after immunoprecipitation (data not shown). However, when transfected with T5, there is a significant amount of HLA-B5 precipitated in the 0.220B5 cells (Figure 6a). The maturation rate of HLA-B5 is approximately the same as in 0.45 , which expresses fulllength tapasin (Figure 6a). Notably, the amount of endoH-resistant HLA-B5 immunoprecipitated from 0.220-T5 begins to decline after $2 \mathrm{~h}$ chase, whereas in 0.45 , the levels remain stable (Figure $6 \mathrm{~b}$ ). We have previously shown that this phenomenon is indicative of suboptimal peptide loading of MHC class I, and suggests that a different repertoire of peptides may be loaded onto HLA-B5 in the presence of the soluble tapasin variant.

To test this possibility, we measured the thermostability of HLA-B5molecules assembled in 0.220-B5T5 compared to those synthesised in 0.45 . We have shown that this assay is a good indicator of the average affinity of peptides bound to a cohort of class I MHC. ${ }^{8,28,29}$ Lysates were precleared for $1 \mathrm{~h}$ at $4{ }^{\circ} \mathrm{C}$ with monoclonal antibody BB7.2 that was previously shown to remove all HLA-A2 molecules (present in 0.45 but not $0.220-B 5 T 5$, data not shown). Figure 7 shows that similar amounts of HLA-B5 were recovered from both cells at $4^{\circ} \mathrm{C}$, but those recovered from 0.220 -B5T5 were less thermostable, with loss of almost half the molecules at $26^{\circ} \mathrm{C}$ and over $60 \%$ loss at $37^{\circ} \mathrm{C}$. These results indicate that although the soluble tapasin restored cell surface expression of HLAB5 and presentation of an immunodominant T-cell epitope, it did not completely restore the loading of optimal peptides capable of stabilising MHC class I at physiological temperatures.

\section{Discussion}

The presence of an incompletely processed tapasinencoding mRNA in several EST databases generated from libraries of diverse cellular and species origin suggested that the T5 transcript with retained introns 5-7 represented an alternatively spliced product and not a processing intermediate isolated from contaminating nuclear RNA. This was confirmed by our ability to detect transcripts that included intron 5 in highly purified preparations of cytosolic mRNA from various sources.

However, upon transfection into the B-cell line 0.220B5, this transcript was processed further to remove intron 5, yielding a transcript that encoded the lumenal domain of tapasin. This is the first time that a soluble tapasin protein has been shown to arise from an mRNA containing all eight exons. Given these observations, it is possible that $\mathrm{T} 5$ represents a naturally occurring intermediate generated during the splicing of tapasin premRNA that is committed to the generation of a soluble isoform of the tapasin protein, but that this is a minor pathway given the low level of detection of 'intron 5-in.' transcripts in normal cells. Our inability to detect this soluble isoform does not imply that it does not naturally exist, as specific cellular signals may be necessary to induce its generation. Such signals may be pathogen derived or relate to specific cell processes, that is, crosspresentation/tolerance. It is possible that the rate 


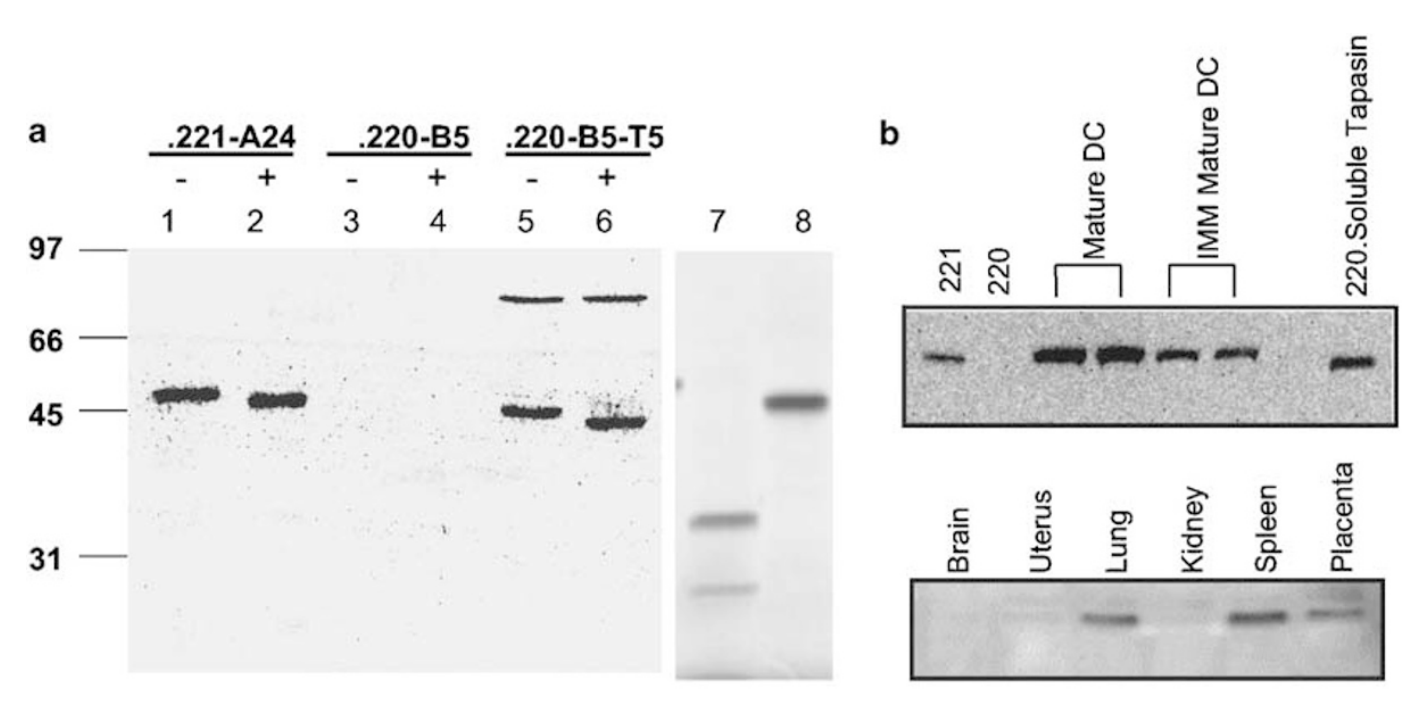

C

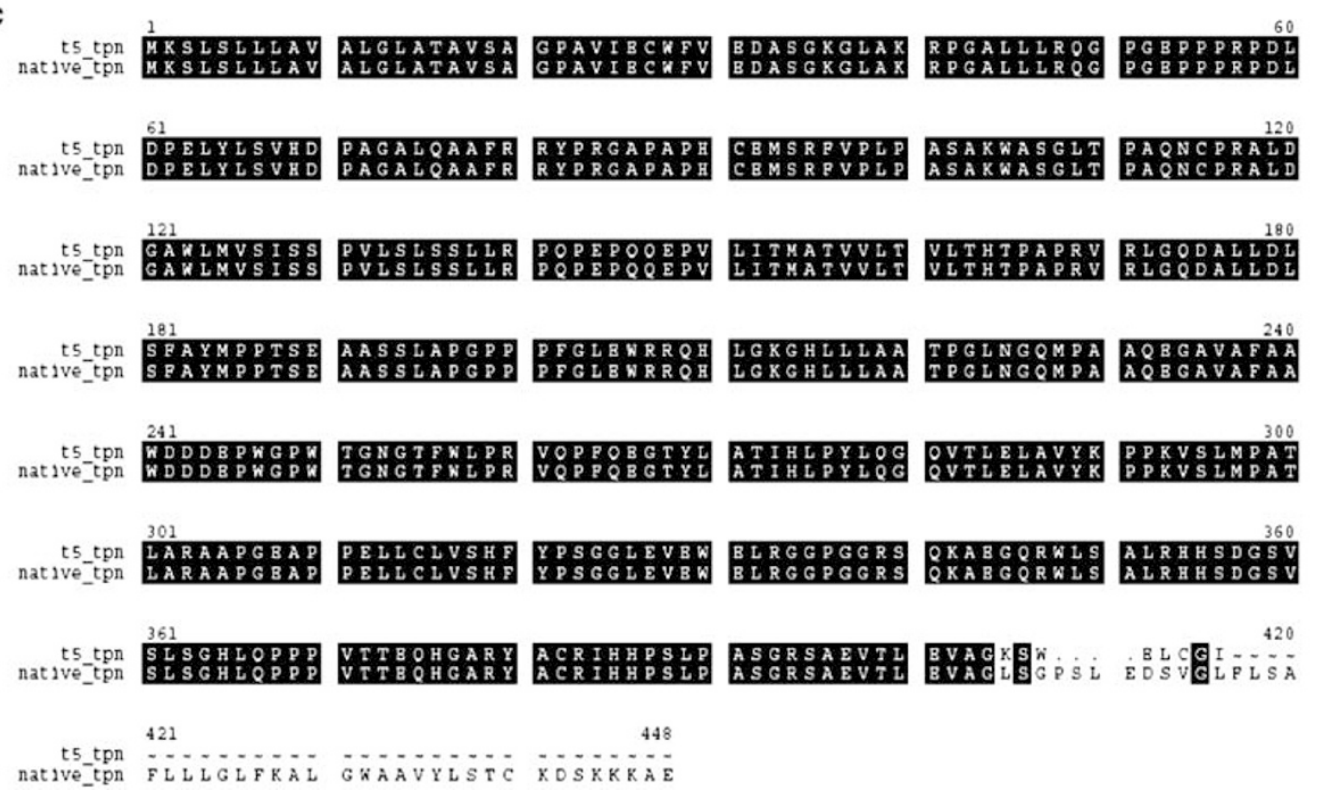

Figure 3 Alternatively spliced tapasin encodes a shorter version of tapasin in 0.220-B5. (a) 0.221-A24 (lanes 1 and 2), or 220 (lanes 3 and 4 ) or 220-B5 (lanes 5 and 6) cells were lysed in 1\% NP-40 and incubated with $(+)$ or without $(-)$ endoglycosidase $\mathrm{H}$ to remove immature N-linked glycans, fractionated by SDS-PAGE and immunoblotted with an antiserum raised to the N-terminus of tapasin. In a separate experiment, T5 cDNA (lane 7) and native tapasin (lane 8) were translated in vitro (as described in Materials and methods section) and the product analysed by Western blotting. (b) Western blot analysis of mature and immature human dendritic cells (top panel) and an array of human tissues (bottom panel) with an antiserum to tapasin. Full-length tapasin expressed in 0.221, and a soluble construct of tapasin having the same molecular size as the processed T5 product expressed in 0.220 are included in the top panel for comparison. (c) Comparison of the predicted amino-acid sequence of the processed T5 transcript and native, full-length tapasin. The additional eight amino acids (KSWELCGI) are seen after amino acid 384 of the mature protein (404 aa from methionine).

at which intron 5 is excised from pre-mRNA relative to other introns (ie the sequence in which the introns are spliced) determines the level of T5-like transcripts, as has been shown for some metazoan genes. ${ }^{30}$ Although we found that the levels of T5-like transcripts in normal lymphocytes did not change upon activation, other cellular changes may affect the alternative splicing of this gene, as has been described for others, including environmental stress, malignant transformation, developmental processes and infection. ${ }^{31}$

We do not know whether the generation of a soluble tapasin will have any physiological significance. The expression of a soluble tapasin in normal cells may modulate the repertoire of peptides that are presented at the cell surface, which may in turn influence the recognition of target cells by CTLs and NK cells. Such codominant expression may be important for preventing the presentation of certain CTL epitopes, while at the same time maintaining good cell surface expression of tapasin-dependent class I MHC molecules that are ligands for NK-inhibitory receptors such as HLA-E. Alternatively, the production of soluble tapasin, which could access intracellular compartments distal to the ER, may be important in assisting peptide loading of MHC class I molecules at these alternative sites. This may have implications for MHC class I crosspresentation within 

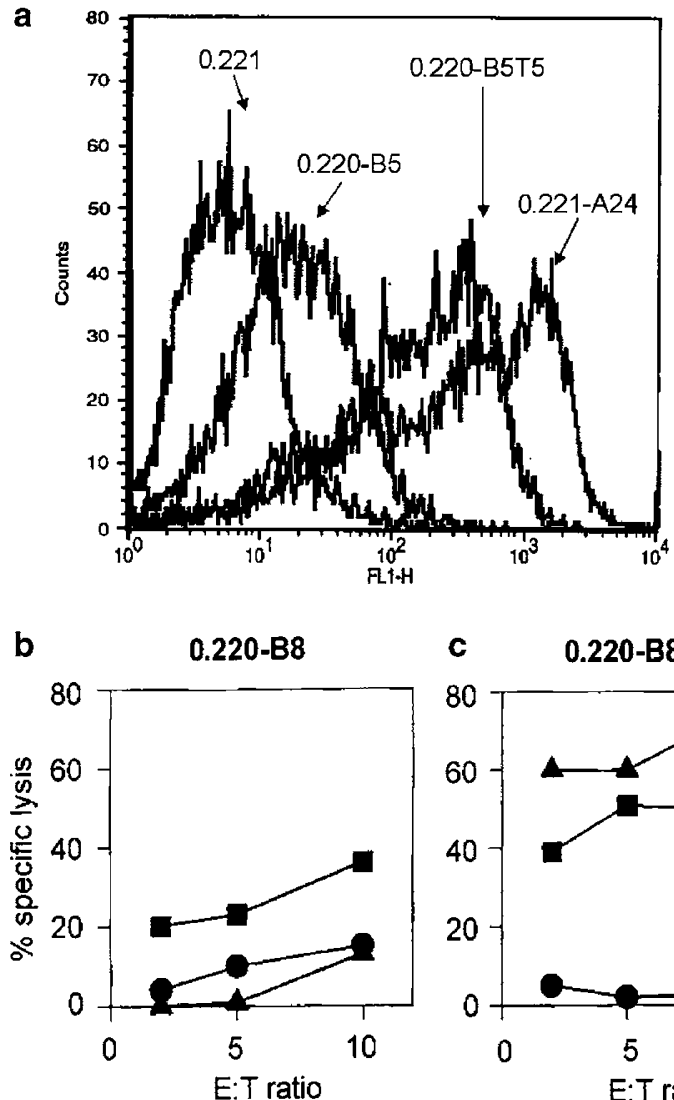

c

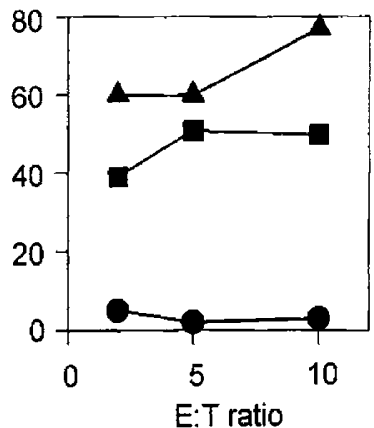

Figure 4 T5 restores normal surface expression of HLA-B5 and rescues CTL recognition. (a) Cell surface expression of MHC class I complexes on $0.221,220-\mathrm{B} 5,221-\mathrm{A} 24$ and $0.220-\mathrm{B} 5-\mathrm{T} 5$ determined by flow cytometry with W6/32. (b) Recognition of 0.220-B8 or 220-B8 transfected with T5 (0.220-B8T5), by HLA-B8restricted anti-HIV-gag CTL, after being infected with HIV-gag-vac (triangle), mock treated (circle) or pulsed with $10 \mu \mathrm{m}$ peptide (square)

\section{Anti-TAP i.p}

0.45

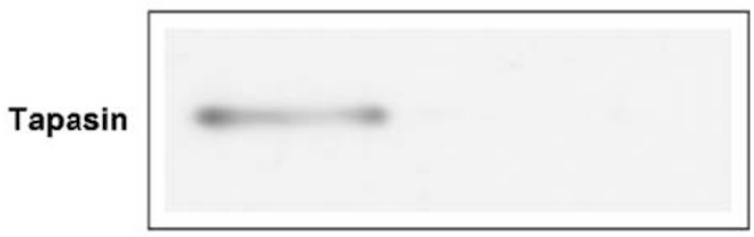

$\beta 2 \mathrm{~m}$

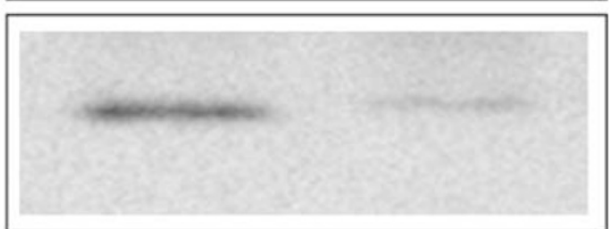

Figure 5 T5-encoded tapasin does not bind to TAP and results in the loss of MHC class I molecules from the PLC. Immunoprecipitates from lysates of $0.220 \mathrm{~B} 5 \mathrm{~T} 5$ and the related (tapasincompetent) line 0.45 were prepared with anti-TAP2 antiserum under conditions that preserve the integrity of the PLC. These were fractionated by SDS-PAGE and immunoblotted with antisera to either tapasin (a) or $\beta 2-\mathrm{m}(\mathbf{b})$ to detect the presence of MHC class I in the complex. a

Chase time (min) $\quad 0 \quad 10 \quad 20 \quad 40 \quad 80 \quad 160$

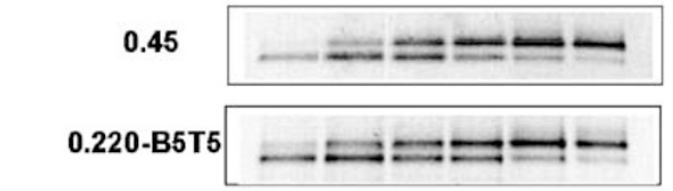

b

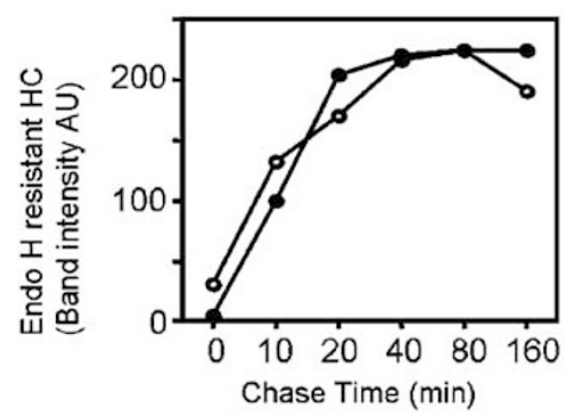

Figure 6 Intracellular maturation of HLA-B5 in T5-transfected 0.220 -B5 cells 0.220 -B5T5 and 0.45 cells were pulse labelled for $10 \mathrm{~min}$ and chased for different time periods as indicated. Lysates were immunoprecipitated with $\mathrm{mAb} \mathrm{W6} / 32$, digested with endo-H and resolved on $12.5 \%$ SDS-PAGE (a), and the band intensity of mature class I HCs (endo-H-resistant) 0.45 (filled circle) and 0.220B5T5 (open circle) was quantitated (b).
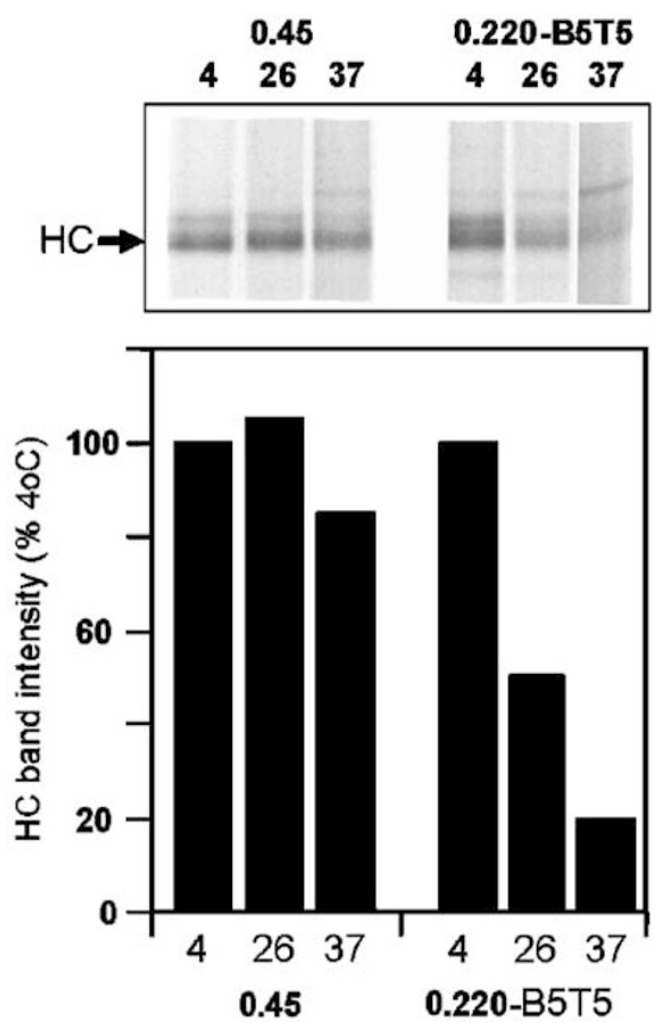

Figure 7 Expressed HLA-B5 in 220-B5T5 is less stable than that in 0.45 , the HLA-B5-expressing wild-type counterpart of 0.220-B5 0.220 -B5T5 and 0.45 cells were labelled for $40 \mathrm{~min}$. After a short preclearing step at $4{ }^{\circ} \mathrm{C}$ (see Materials and methods section), the supernatants were incubated for $1 \mathrm{~h}$ at $4^{\circ} \mathrm{C}, 26^{\circ} \mathrm{C}$ or $37^{\circ} \mathrm{C}$ as indicated, before recovering assembled HLA-B5 with mAb W6/32. The class I HCs were resolved by SDS-PAGE (top panel) and quantified by autoradiography (bottom panel). 
compartments such as endosomes or the MIIC, where MHC class I complexes have been previously identified and investigated for peptide editing and exchange. ${ }^{32-34}$

\section{Materials and methods}

Cells and antibodies

All cells were cultured in RPMI 1640 supplemented with $10 \%$ FCS, glutamine and antibiotics 721.220 (0.220), $721.221(0.221)$ and their respective HLA transfectants, 0.220-B5, 0.220-B8, 0.221-A24 and HLA-B5-expressing hemizygous cell 721.45, were kind gifts from $\mathrm{R}$ DeMars (University of Wisconsin). Human DC cell lines were derived from CD14-positive monocytes as described previously. ${ }^{35}$ PBLs were isolated from whole blood by standard percol centrifugation and maintained in culture overnight. The monoclonal antibody W6/32 was purified by standard protein A affinity chromatography. The antibody recognises a conformation-sensitive, monomorphic epitope on HLA-A, $-\mathrm{B}$ and $-\mathrm{C}$, and requires that the $\mathrm{HC}$ be assembled with $\beta 2-\mathrm{m} .{ }^{36}$ The antitapasin antibody (T66) was raised in rabbits against the first 62 amino acids of mature tapasin fused to GST protein using pGEX vector (Pharmacia). The anti-TAP2 antiserum was a gift from J Neefjes (Netherlands Cancer Institute). Rabbit anti-calreticulin antiserum was purchased from Affinity Bioreagents Inc. BBM1.1 was purified by standard protein A affinity chromatography and recognises human $\beta 2-\mathrm{m} .{ }^{37}$

\section{Alternatively spliced tapasin gene subcloning}

cDNAs obtained from UK HGMP resource Centre (Cambridge, UK) based on their sequence homology to tapasin were sequenced both manually and automatically using an ABI sequencer. For transfections, the EST clone corresponding to exons 1-8 containing introns 5-7 (T5) was subcloned into pcDNA 3.0. Clones were sequenced to ensure correct orientation.

\section{Transfection}

A total of $1 \times 10^{7} 0.220$-B5 cells were washed twice with $15 \mathrm{ml}$ of phosphate-buffered saline (PBS), resuspended in $0.9 \mathrm{ml}$ of PBS and then mixed with $100 \mu \mathrm{l}$ of pcDNA3 containing the T5 fragment in $0.4 \mathrm{~cm}$ electrode gap curvettes (Bio-Rad) and kept on ice for $20 \mathrm{~min}$. The electroporation was performed using the Gene Pulser (BioRad) set at $210 \mathrm{~V}$ and $960 \mu \mathrm{F}$. Stable transfectants were selected with $500 \mu \mathrm{g} / \mathrm{ml}$ G418 (Life Technology) and screened for MHC class I expression by FACS. The positive cell lines were subcloned by limited dilution and clones were selected for further analysis based on their cell surface expression of MHC class I.

\section{Pulse chase and immunoprecipitation}

A total of $1 \times 10^{7}$ cells were starved in $4 \mathrm{ml}$ of cysteineand methionine-deficient RPMI 1640 (Sigma) with 10\% dialysed FCS at $37^{\circ} \mathrm{C}$ for $30 \mathrm{~min}$. The cells were spun down and resuspended in $0.5 \mathrm{ml}$ of the same solution containing $200 \mu \mathrm{Ci}$ Promix (Amersham) and incubated at $37^{\circ} \mathrm{C}$ for a further $10 \mathrm{~min}$. The labelled cells were washed with $20 \mathrm{ml}$ of cold PBS and immediately lysed in $1 \mathrm{ml}$ of TBS containing $1 \%$ digitonin, protease inhibitor cocktail (Sigma), and then kept on ice for $30 \mathrm{~min}$. After removing nuclei by centrifugation at 13.00 r.p.m. for $30 \mathrm{~min}$, the cell lysates were precleared by incubation at $4{ }^{\circ} \mathrm{C}$ either overnight (or for $20 \mathrm{~min}$ in the case of thermostability assays) with $1 \%$ normal rabbit serum (Sigma) and $50 \mu \mathrm{l}$ of prewashed $(0.1 \%$ digitonin) Staph A (Sigma). For immunoprecipitation of HLA-B5 with $\mathrm{mAb}$ W6/32, preclearing with $\mathrm{mAb}$ BB7.2 was performed. Antibodies were added to precleared supernatants for $90 \mathrm{~min}$ before adding $50 \mu \mathrm{l}$ of Protein A-Sepharose 4B (Sigma) and rotated at $4{ }^{\circ} \mathrm{C}$ for further $30 \mathrm{~min}$. Immunoprecipitates were resolved in $12.5 \%$ SDS-PAGE. Immunoglobulin bands were visualised with Coomassie blue to confirm equivalent loading of each lane. Gels were then soaked in amplify (Pharmacia) for $15 \mathrm{~min}$, dried and exposed to an $X$-ray film overnight. Alternatively, gels were processed for immunoblotting (see below)

\section{In vitro translation}

Transcription and translation were carried out simultaneously using TNT Quick coupled Transcription/translation systems (Promega) according to the manufacturer's instructions. Briefly, $1 \mu \mathrm{g}$ of plasmid was mixed with $40 \mu \mathrm{l}$ of master mix and $20 \mu \mathrm{Ci}$ of Promix (Amersham) and nuclease-free water was added to a final volume of $50 \mu \mathrm{l}$. The reaction was carried out at $30^{\circ} \mathrm{C}$ for $90 \mathrm{~min}$

\section{Immunoblotting}

Proteins were transferred from polyacrylamide gels to Hybond membrane (Amersham) electrophoretically over $90 \mathrm{~min}$ at $25 \mathrm{~V}$. After blocking membranes with $2.5 \%$ skimmed milk in PBS, the membrane was incubated with anti-tapasin antiserum (1:10000 dilution) in $2.5 \%$ skimmed milk/0.2\% Tween-20 PBS for at least $1 \mathrm{~h}$ at room temperature. The membrane was washed with PBS/Tween-20 twice and incubated with anti-rabbit antibody conjugated to horseradish peroxidase. The membrane was thoroughly washed with PBST and visualised with an enhanced chemiluminescence detection system according to the manufacturer's instructions (Amersham). A multi-human tissue protein array (BioChain Institute, Hayward, CA, USA) was probed with tapasin antiserum (1:5000 dilution) and anti-rabbit antibody conjugated to horseradish peroxidase (as above).

\section{RT-PCR}

mRNA was extracted from $1 \times 10^{6}$ cells using an mRNA Direct kit (Dynal). cDNA was synthesised using standard random priming methods with MuMLV reverse transcriptase (Promega) and RNAse inhibitor (Promega). RT-PCR was carried out with the primers ATGGGG CACGCTATGCCTGT as the forward primer and CT ACGCTGTCCTCAAGGGAG as the reverse primer. Amplification was carried out as follows: $4 \mathrm{~min}$ at $94^{\circ} \mathrm{C}$, followed by 30 cycles of: $94^{\circ} \mathrm{C}$ for $30 \mathrm{~s}, 45^{\circ} \mathrm{C}$ for $30 \mathrm{~s}, 72^{\circ} \mathrm{C}$ for $1 \mathrm{~min}$ and final extension for $8 \mathrm{~min}$ at $72^{\circ} \mathrm{C}$.

\section{FACS analysis}

In all, $2 \times 10^{5}$ cells were incubated with a final concentration of $10 \mu \mathrm{g} / \mathrm{ml} \mathrm{W6/32}$ in RPMI 1640 with $10 \%$ FCS $(10 \mu \mathrm{g} / \mathrm{ml})$ at $4^{\circ} \mathrm{C}$ for one hand washed with $10 \mathrm{ml}$ cold PBS twice. The cells were stained for $45 \mathrm{~min}$ at $4{ }^{\circ} \mathrm{C}$ in $0.5 \mathrm{ml}$ PBS containing a 200-fold dilution of goat antimouse Ig-FITC conjugate (Sigma). The cells were washed with $0.5 \mathrm{ml}$ of cold PBS three times and suspended in $0.3 \mathrm{ml}$ of PBS containing $0.7 \%$ formaldehyde. The 
samples were analysed in a Becton-Dickinson Flow Cytometer with minimum 20000 cells counted.

\section{CTL assays}

Target cells were labelled with ${ }^{51} \mathrm{Cr}$ for $90 \mathrm{~min}$ after being infected with recombinant vaccinia virus at a multiplicity of five overnight at $37^{\circ} \mathrm{C}$. After washing, the cells were exposed to CTL in a standard 4-h Chromium release assay. For peptide-sensitised targets, $10 \mu \mathrm{M}$ peptide was included in CTL assay.

\section{Acknowledgements}

We would like to thank G Screaton for helpful discussion. This work was supported by the Wellcome Trust. APW is a Wellcome Clinical Training Fellow.

\section{References}

1 Williams A, Peh CA, Elliott T. The cell biology of MHC class I antigen presentation. Tissue Antigens 2002; 59: 3-17.

2 Princiotta MF, Finzi D, Qian SB et al. Quantitating protein synthesis, degradation, and endogenous antigen processing. Immunity 2003; 18: 343-354.

3 Schubert U, Anton LC, Gibbs J, Norbury CC, Yewdell JW, Bennink JR. Rapid degradation of a large fraction of newly synthesized proteins by proteasomes. Nature 2000; 404: 770-774.

4 Sadasivan B, Lehner PJ, Ortmann B, Spies T, Cresswell P. Roles for calreticulin and a novel glycoprotein, tapasin, in the interaction of MHC class I molecules with TAP. Immunity 1996; 5: 103-114.

5 Ortmann B, Androlewicz MJ, Cresswell P. MHC class I/beta 2microglobulin complexes associate with TAP transporters before peptide binding. Nature 1994; 368: 864-867.

6 Ortmann B, Copeman J, Lehner PJ et al. A critical role for tapasin in the assembly and function of multimeric MHC class I-TAP complexes. Science 1997; 277: 1306-1309.

7 Gao B, Adhikari R, Howarth $\mathrm{M}$ et al. Assembly and antigenpresenting function of MHC class I molecules in cells lacking the ER chaperone calreticulin. Immunity 2002; 16: 99-109.

8 Dick TP, Bangia N, Peaper DR, Cresswell P. Disulfide bond isomerization and the assembly of MHC class I-peptide complexes. Immunity 2002; 16: 87-98.

9 Serwold T, Gonzalez F, Kim J, Jacob R, Shastri N. ERAAP customizes peptides for MHC class I molecules in the endoplasmic reticulum. Nature 2002; 419: 480-483.

10 Saric T, Beninga J, Graef CI, Akopian TN, Rock KL, Goldberg AL. Major histocompatibility complex class I-presented antigenic peptides are degraded in cytosolic extracts primarily by thimet oligopeptidase. J Biol Chem 2001; 276: 36474-36481.

11 Brocke P, Garbi N, Momburg F, Hammerling GJ. HLA-DM, HLA-DO and tapasin: functional similarities and differences. Curr Opin Immunol 2002; 14: 22-29.

12 Bangia N, Lehner PJ, Hughes EA, Surman M, Cresswell P. The N-terminal region of tapasin is required to stabilize the MHC class I loading complex. Eur J Immunol 1999; 29: 1858-1870.

13 Hughes EA, Cresswell P. The thiol oxidoreductase ERp57 is a component of the MHC class I peptide-loading complex. Curr Biol 1998; 8: 709-712.

14 Williams AP, Peh CA, Purcell AW, McCluskey J, Elliot T. Optimization of the MHC class I peptide cargo is dependent on tapasin. Immunity 2002; 16: 509-520.

15 Tan P, Kropshofer H, Mandelboim O, Bulbuc N, Hammerling GJ, Momburg F. Recruitment of MHC class I molecules by tapasin into the transporter associated with antigen processing-associated complex is essential for optimal peptide loading. J Immunol 2002; 168: 1950-1960.

16 Chun T, Grandea III AG, Lybarger L, Forman J, Van Kaer L, Wang CR. Functional roles of TAP and tapasin in the assembly of M3- $N$-formylated peptide complexes. J Immunol 2001; 167: 1507-1514.

17 Park B, Ahn K. An essential function of tapasin in quality control of HLA-G molecules. J Biol Chem 2003.

18 Peh CA, Burrows SR, Barnden M et al. HLA-B27-restricted antigen presentation in the absence of tapasin reveals polymorphism in mechanisms of HLA class I peptide loading. Immunity 1998; 8: 531-542.

19 Garbi N, Tan P, Diehl AD et al. Impaired immune responses and altered peptide repertoire in tapasin-deficient mice. Nat Immunol 2000; 1: 234-238.

20 Grandea III AG, Golovina TN, Hamilton SE et al. Impaired assembly yet normal trafficking of MHC class I molecules in tapasin mutant mice. Immunity 2000; 13: 213-222.

21 Herberg JA, Sgouros J, Jones T et al. Genomic analysis of the tapasin gene, located close to the TAP loci in the MHC. Eur J Immunol 1998; 28: 459-467.

22 Lehner PJ, Surman MJ, Cresswell P. Soluble tapasin restores MHC class I expression and function in the tapasin-negative cell line 0.220. Immunity 1998; 8: 221-231.

23 Turnquist HR, Vargas SE, Reber AJ et al. A region of tapasin that affects L(d) binding and assembly. J Immunol 2001; 167: 4443-4449.

24 Altschul SF, Madden TL, Schaffer AA et al. Gapped BLAST and PSI-BLAST: a new generation of protein database search programs. Nucleic Acids Res 1997; 25: 3389-3402.

25 Copeman J, Bangia N, Cross JC, Cresswell P. Elucidation of the genetic basis of the antigen presentation defects in the mutant cell line 0.220 reveals polymorphism and alternative splicing of the tapasin gene. Eur J Immunol 1998; 28: 3783-3791.

26 Greenwood R, Shimizu Y, Sekhon GS, DeMars R. Novel allelespecific, post-translational reduction in HLA class I surface expression in a mutant human B cell line. J Immunol 1994; 153: $5525-5536$.

27 Park B, Lee S, Kim E, Ahn K. A single polymorphic residue within the peptide-binding cleft of MHC class I molecules determines spectrum of tapasin dependence. J Immunol 2003; 170: $961-968$

28 Dedier S, Reinelt S, Reitinger T, Folkers G, Rognan D. Thermodynamic stability of HLA-B*2705. Peptide complexes. Effect of peptide and major histocompatibility complex protein mutations. J Biol Chem 2000; 275: 27055-27061.

29 Tan L, Andersen MH, Elliott T, Haurum JS. An improved assembly assay for peptide binding to HLA-B 2705 and $\mathrm{H}-2 \mathrm{~K}(\mathrm{k})$ class I MHC molecules. I Immunol Methods 1997; 209: 25-36.

30 Patel AA, McCarthy M, Steitz JA. The splicing of U12-type introns can be a rate-limiting step in gene expression. EMBO J 2002; 21: 3804-3815.

31 Faustino NA, Cooper TA. Pre-mRNA splicing and human disease. Genes Dev 2003; 17: 419-437.

32 Gromme M, Uytdehaag FG, Janssen $\mathrm{H}$ et al. Recycling MHC class I molecules and endosomal peptide loading. Proc Natl Acad Sci USA 1999; 96: 10326-10331.

33 Castellino F, Boucher PE, Eichelberg K et al. Receptormediated uptake of antigen/heat shock protein complexes results in major histocompatibility complex class I antigen presentation via two distinct processing pathways. J Exp Med 2000; 191: 1957-1964.

34 Kleijmeer MJ, Escola JM, UytdeHaag FG et al. Antigen loading of MHC class I molecules in the endocytic tract. Traffic 2001; 2 124-137.

35 Pickl WF, Majdic O, Knapp W. Dendritic cell generation from highly purified CD14+ monocytes, in Robinson SP, Stagg AJ (eds). Dendritic Cell Protocols. Humana Press: NJ, 2001, pp 283-303.

36 Parham P, Barnstable CJ, Bodmer WF. Use of a monoclonal antibody (W6/32) in structural studies of HLA-A, B, C, antigens. J Immunol 1979; 123: 342-349.

37 Parham P, Androlewicz MJ, Holmes NJ, Rothenberg BE. Arginine 45 is a major part of the antigenic determinant of human beta 2-microglobulin recognized by mouse monoclonal antibody BBM.1. J Biol Chem 1983; 258: 6179-6186. 